\title{
Herman Dooyeweerd und das «reformierte» Naturrecht
}

\author{
Johannes Corrodi
}

Stephen J. Grabills Buch «Rediscovering the Natural Law in Reformed Theological Ethics» trägt bei zu einem anschwellenden Fluss wissenschaftlicher Publikationen, die sich dem Thema des Naturrechts aus historischer und reformiert-theologischer Sicht stellen. ${ }^{1}$ Grabill et al. geht es dabei um nichts weniger als die kritische Revision einer bestimmten, spätestens seit 1934, dem Jahr der Barth-Brunner Debatte, selbst «scholastisch" gewordenen Perspektive auf die Reformation, derzufolge der Bruch mit dem mittelalterlichscholastischen Denken in Kirche und Theologie die ständig gefährdete Errungenschaft einiger überlebensgrosser Persönlichkeiten war. Charakteristisch für diese Perspektive sei der Versuch, den ursprünglich evangelischen Glauben gegen die Vereinnahmung durch ein mit "Rom» assoziiertes, naturrechtlich-philosophisches Denken zu schützen. Der Gestus der Abgrenzung verdunkle jedoch die Anerkennung der historischen Einflüsse früherer Denker, allen voran T. von Aquin und J. Duns Scotus, sowie der Kontinuitäten im naturrechtlichen Denken innerhalb der reformierten Theologie bis 1700 . Wo diese Themen dennoch aufgegriffen und gewürdigt würden, greife man auf ein allzu verbreitetes Interpretationsmuster zurück, dem gemäss nachfolgende «orthodoxe» Theologen eben nicht die Statur und Weitsicht eines J. Calvin oder M. Luther gehabt hätten und deshalb die zähen Banden der naturrechtlichen Tradition(en) nicht vollständig abzuschütteln in der Lage gewesen seien oder diese gar freiwillig erneuerten.

Paradigmatisch sei das Urteil K. Barths, dass zumindest bei Calvin selbst die formale Möglichkeit, geschweige denn die faktischen Überreste einer Naturrechtstheorie ausgeschlossen werden könnten. ${ }^{2}$ Dieses apodiktische Urteil, kombiniert mit der Prämisse, dass "Calvin as the chief codifier and lodestar of Reformed doctrine» ${ }^{3}$ zu gelten habe, sei eine der zentralen Ursache für das Ausbleiben einer fundierten wissenschaftlichen Erforschung und Analyse des Naturrechtdenkens im reformierten Kontext. Umgekehrt werde

1 S.J. Grabill, Rediscovering the Natural Law in Reformed Theological Ethics, Grand Rapids 2006.

2 Ebd., 71, 85.

3 Ebd., 3 . 
katholischerseits oft immer noch das historisch ebenso schwer aufrechtzuerhaltende Stereotyp einer intrinsischen Verbindung zwischen reformatorischem und nominalistisch-voluntaristischem Denken stark gemacht, so dass Gottes Souveränität nicht von der Willkürherrschaft eines himmlischen Tyrannen unterschieden werden könne, ein Umstand der dem Staatsabsolutismus eines T. Hobbes oder noch Schlimmerem Vorschub geleistet hätte. ${ }^{4}$

Grabill kann auf eine eindrückliche historische Forschung und Gelehrsamkeit zurückgreifen, um die Kontinuitätsthese in Sachen reformiertem Naturrechtsdenken zu stützen, die seit den 1990er Jahren zumindest wieder diskutabel geworden sei. Doch ist die Fülle des von ihm angeführten Materials umgekehrt proportional zur Reflexion der Frage, worin denn die erhoffte Bedeutung einer breiteren Anerkennung dieser These bestehen soll. Die Antwortrichtung lässt sich einigen wenigen Stellen entnehmen. Es geht um die Wiederbelebung der "grand moral tradition" ${ }^{5}$, die ein Aristoteles, Cicero, Augustinus, T. von Aquin, Duns Scotus und nun eben auch Calvin, P. Martyr Vermigli, G. Zanchi, J. Althusius, F. Turretin, Ch. und A. A. Hodge und viele andere mehr verbinde. ${ }^{6}$ Die Betonung einer gemeinsamen natürlich-moralischen Basis soll dem Aufbau und der Pflege von Gemeinschaft, die durch ein Durcheinander von religiösen und säkularistischen Partikularinteressen bedroht sei, dienen (der gegenwärtige nordamerikanische Kontext des Autors muss hier mitbedacht werden). An diesem Punkt scheint keine weitere Begründung mehr nötig. Gemeinschaft entsteht durch die Anerkennung des Gemeinsamen. Theologisch gewendet: die Geltung der Sprache der Schöpfung und der natürlichen Vernunft sei unabhängig von der spezifisch christlichen Gotteserfahrung und ihrem konfessionellen Artikulationsspektrum, vermutlich inklusive agnostischer oder «freidenkerischer» Negationsformen. Wohlgemerkt wird hier nicht der kantisch-liberale Gemeinplatz bemüht, die Sprache des Glaubens sei eben nicht «allgemein zugänglich» und müsse deshalb in ein (ur- oder ausser-?)menschliches Idiom der Vernunft oder eines ihrer gesellschaftlichen Platzhalter übersetzt werden. Vielmehr unterstreicht Grabill die Tatsache, dass für Calvin nur die Undankbarkeit jemanden dazu bringen könne, die natürliche Erkenntnis Gottes extra Christum, "woanders» zu suchen. ${ }^{7}$ Was aber nicht bedeute, dass von dieser Erkenntnis nichts

\footnotetext{
Ebd., 56.

Ebd., 14

Ebd., 19

Ebd., 85-86.
} 
übrig geblieben sei, oder sie nicht konstruktiv-gemeinschaftsstiftend eingesetzt werden könne.

Unter den von Grabill kritisierten Denkern, die sich einer naturrechtlich induzierten kommunikativen Einheit widersetzen, ist auch der niederländische Philosoph Herman Dooyeweerd (18941977) zu finden. Das ist zunächst nicht erstaunlich. Der in der Wolle gefärbte (Neo-)Calvinist, der zudem auch noch profilierter Rechtstheoretiker war, vertrat in reiferen Jahren eine deutlich anders ausgerichtete Auffassung der Bedeutung des Rechts auf all seinen Ebenen. Ursprünglich selbst dem naturrechtlichen Denken nahe, findet Dooyeweerd in der Idee des Gesetzes den Ankerpunkt seines philosophischen Denkens schlechthin. Nicht zufällig ist sein Ansatz als die Wijsbegeerte der Wetsidee, Philosophie der Gesetzesidee oder Philosophy of the Cosmonomic Idea bekannt geworden. ${ }^{8}$ Es ist deshalb etwas erstaunlich, dass Grabill in Dooyeweerd einen weiteren reformierten Theologen zu erkennen meint, der jeden religiös motivierten Willen zu Gestaltung und theoretischer Durchdringung der «weltlichen» Gesellschaftsordnung für gleichbedeutend mit der Selbstabschaffung des genuin «reformierten» theologischen Denkens hält.

Von der geteilten Ablehnung seiner avisierten (Neu-)Ausrichtung reformierten Denkens einmal abgesehen, entbehrt Grabills Zusammenschluss von Namen wie K. Barth, G.C. Berkouwer, H. Dooyeweerd und C. Van Til denn jeder sachlichen Berechtigung. ${ }^{9}$ Wenn die überhöhten Erwartungen an eine gemeinsame moralischrechtliche Grundlage - ob nun religiös oder säkular begründet post lapsum mit untrüglicher Sicherheit zu neuem Streit über eben diese Grundlagen führen, so muss an dieser Stelle an die umgekehrte

\footnotetext{
8 Es gibt zwei Texte von H. Dooyeweerd in deutscher Sprache, davon der eine in diesem Rahmen von besonderem Interesse ist, da er erstens vom Naturrecht handelt und zweitens auf einem Vortrag beruht, der «für die theologische Konferenz in Zürich am 28. Februar 1939" gehalten wurde (H. Dooyeweerd, Das natürliche Rechtsbewusstsein und die Erkenntnis des geoffenbarten göttlichen Gesetzes, in: Antirevolutionaire Staatkunde 13.Jg., 1939, 157-182,157). Die einzige umfassende Publikation in deutscher Sprache zu H. Dooyeweerd ist derzeit J. Schick, Denken des Ganzen. Eine vergleichende Studie zu den Wirklichkeitsanschauungen Karl Heims und Herman Dooyeweerds angesichts der Herausforderungen durch Postmoderne und neue Metaphysik, Göttingen 2006. Eine gute Einführung in das rechtliche und politische Denken Dooyeweerds gibt J. W. Skillen, Philosophy of the Cosmonomic Idea: Herman Dooyeweerd's Political and Legal Thought, The Political Science Reviewer 32, 2003, 318-380. Für die wichtigsten bio-bibliographischen Daten siehe BiographischBibliographisches Kirchenlexikon: http://www.bbkl.de/d/dooyeweerd_h.shtml (eingesehen 31. Mai 2006)

9 Grabill, Rediscovering, s. Anm. 1, 175, 187.
} 
Binsenwahrheit erinnert werden: Wer gemeinsame Gegner hat, ist nicht schon deswegen Freund. ${ }^{10}$ Durch den mangelnden Willen zur Differenzierung verbaut sich Grabill nun den kritischen Zugang zu einer zentralen Frage, die Dooyeweerd schon früh umgetrieben, und schliesslich zur Abkehr vom naturrechtlichen Denken in seinen religiösen und säkularen Spielarten bewogen hat. Wie gesagt, Dooyeweerds Antwort auf die Frage ist nicht unbedingt dazu angetan, die Sympathien der genannten «Mitstreiter» zu evozieren.

Die Frage selbst ist leicht gestellt: Gründet Recht in moralischen Prinzipien der Vernunft? Wenn ja, wie? Die von Grabill angeführte "grand tradition" hatte keinen Zweifel, dass dem so ist. Auf dem Grund der menschlichen Vernunft spiegelt sich das ewige Gesetz, das dem Urheber und Lenker des Kosmos vor Anbeginn dieser Welt als Schöpfungsvorlage diente. In den Worten von J. Skillen:

«Aquinas, as Dooyeweerd interprets him, sometimes blurs the boundary between God and the creation with respect to both law and reason and tends to reduce or condense all modal normativity to rationality. Natural law is the sharing in eternal law by intelligent creatures; the relationships between men> and «of men to God` are subject to the dictates of natural law, which represents the sharing of human intelligence in the eternal law. The creation's social diversity coheres in rational lawfulness - both divine law and natural law, which also serve as moral norm. Human reason thus, to some degree, appears to be both subject and law at the same time.» ${ }^{11}$

Die grundlegende Idee ist die einer Art Gemeinschaft aller vernunftbegabten Wesen, einschliesslich Gott. Darüber herrscht Einhelligkeit. Die Geschichte des Zerfalls dieser Einhelligkeit lässt sich nun auf verschiedene Weise erzählen. Tatsache jedoch ist, dass sie verschwunden ist. «Might is Right» lautet die unbeschönigte Kurzformel eines unterschwelligen nach-idealistischen Gegenkonsenses, der die normativen Grundlagen des Rechts eben nicht in moralisch-rationalen, sondern in biotischen, psychischen, historischen, ökonomischen, etc. «Gesetzen» erblicken will. Ein naturalistischer Fehlschluss? Mag sein, doch wird sich von dem Einwand nur beirren lassen, wer von einer ursprünglichen Gesetzes- und Ordnungskraft der Vernunft ausgeht

\footnotetext{
${ }^{10}$ Dooyeweerds Kritik an K. Barth kann in seinem Hauptwerk, der New Critique of Theoretical Thought, Lewiston/Queenston/Lampeter 1997, Band II, 300 ff verfolgt werden.

11 Skillen, Philosophy of the Cosmonomic Idea, s. Anm. 8, 330.
} 
- was nun gerade zum Kernbestand des «überwundenen», religiösen wie säkularen, Naturrechtsdenkens gehört.

Die einseitige Orientierung an einem mathematisch-mechanischen Wissenschaftsideal und der entfesselte humanistische Glaube an die Möglichkeit einer rationalen Neuorganisation von Natur und Gesellschaft anhand einer verbesserten Schöpfungsvorlage hat nach Dooyeweerd letztlich zum Umschlag ins Gegenteil der erhofften Weltordnung gefuihrt. «Unlimited competition on the market made the Hobbesian picture of the state of nature, as a condition of homo homini lupus», into a terrible reality.» ${ }^{12}$

Der Verabsolutierung einer vermeintlich «autonom» gewordenen mathematisch-schöpferischen Vernunft und ihrer nachfolgenden Entmachtung durch die verschiedenen «-ismen» des 19. und 20. Jahrhunderts hält Dooyeweerd das biblisch inspirierte, theonome Prinzip der Sphärensouveränität entgegen.Von A. Kuyper (1837-1920) zu theologischer Prägnanz und gesellschaftspolitischer Wirksamkeit erhoben, soll dieses Prinzip die wechselseitige Irreduzibilität und Kohärenz aller bereichsspezifischen Schöpfungsnormen gewährleisten. Deren Geltung ist nun weder metaphysisch-realistisch an eine lex aeterna, der selbst die göttliche arche $\bar{e}$ vor Anbeginn der Zeiten $\mathrm{zu}$ folgen gehabt hätte, noch nominalistisch-voluntaristisch an eine lex naturalis, die scheinbar direkt einem arbiträren Dekret Gottes entspringt, gebunden. ${ }^{13}$ Im Licht dieser «kosmonomischen Idee» wurzeln alle möglichen Typen von Gesetzmässigkeit und Normativität in der unauslotbaren, pleromatischen Güte des göttlichen Schöpfungswillens und haben deshalb gleichursprünglichen Charakter. Rechtsbezogene Schöpfungsnormen sind also nicht weniger normativ oder integral als ethische, logische, ästhetische, historische oder ökonomische. Das calvinistische Zentralmotiv der Souveränität Gottes deutet Dooyeweerd gerade so, dass sich jede gesellschaftliche und kulturelle Sphäre nach ihren eigenen, historisch-progressiv zu erschliessenden Gesetzmässigkeiten entfalten kann und in diesem Sinn «gottesunmittelbar» ist. Damit ist z.B. einer Nivellierung der Zivilgesellschaft durch den Betreuerstaat - verstanden als «societas perfecta» oder wohlfahrtsorientierte Wertegemeinschaft - wie auch der kontraktualistischen Individualisierung und Atomisierung durch die ebenso totalisierende Logik des Marktes ein prinzipieller Riegel geschoben.

12 Dooyeweerd, New Critique III, s. Anm. 10, 596.

13 Sie lässt sich auch nicht kreuzestheologisch-soteriologisch aushebeln, da selbst die gerechtfertigte, gottferne Kreatur ihre conditio nur als Entstellung eines originären geschöpflichen Sinn-, Ordnungs- oder Lebenszusammenhangs deuten kann. 
Während im Denken Kuypers das Prinzip der Sphärensouveränität noch nicht ausreichend von einer "tautologischen Differenzierung» unterschieden war - so dass Moral $=$ Moral, Politik $=$ Politik, Markt $=$ Markt - argumentiert Dooyeweerd dahingehend, dass jedes «Ding», Ereignis, Prozess, soziale Körperschaft ... in allen modalen Aspekten, d.h. in allen kreatürlichen Gesetzessphären oder Normativitätstypen gleichzeit funktioniert. Nichts in der Welt kann auf eine vermeintliche, aus seiner vor-theoretischen Wesensfülle abstrahierte «Substanz» reduziert werden. Denn Einheit und Zusammenhang der modalen Normen und der durch sie ermöglichten Individualitätsstrukturen ist begründet in der Einheit der Schöpfung als gefallenes und in Jesus Christus in Gemeinschaft mit dem heiligen Geist wiederhergelltes Sinnganzes. Dieses «Ganze» geht in keinem hierarchischen Konstruktionsprinzip - sei dies nun transzendental-logischer oder metaphysisch-organischer Natur - auf. Der irdische Gesetzgeber braucht demnach keine «höheren» Prinzipien zu befolgen um rechtmässigen Gebrauch von seiner Macht zu machen, wenn diese denn in seiner modal-jural bestimmten Autorität gründet. ${ }^{14}$ Die innere Natur dieser Autorität im unauflöslichen Kontext anderer gesellschaftlicher Kompetenzsphären juridisch zu erschliessen ist gerade die Aufgabe einer am Prinzip der Sphärensouveränität orientierten Rechtstheorie und -philosophie. Auf diesem Hintergrund ist es bestenfalls irreführend, wenn die Normativität von Recht, Moral, Wissenschaft, Kunst ... in einer zentralhierarchischen Ordnung von rationalen Prinzipien oder Lebensgütern gesucht wird.

Nicht dass die verstärkte Anziehungskraft naturrechtlicher Denkformen unnachvollziehbar wäre. Wenn die aristotelisch-thomistische Tradition «Recht» und «Gerechtigkeit» unter die "moralischen» Tugenden subsumierte, schuf sich in Nachrenaissancezeiten das Bewusstsein derVerschiedenheit und Einzigartigkeit von Recht und Moral immer grössere Bahn. Grabill liegt vermutlich nicht ganz falsch, wenn er den Namen H. Grotius in Zusammenhang mit dem modernen Auseinandertreten von Recht und Moralität bringt. ${ }^{15}$ Man könnte auch noch J. J. Rousseau erwähnen, wo die absolute Freiheit der Moralität und der absolute Zwang des Gesetzes sich gegenseitig unterlaufen, indem erstere in letzterem begründet sein soll. Doch das Dilemma einer ethischen Politik vs. einer «reinen»

\footnotetext{
${ }_{14}$ Die immer wieder anzutreffende Rede einer notwendigen Beschränkung der politischen Macht gewisser gesellschaftlicher Akteure (inkl. Staat) verdeckt das Problem, wie denn eine durch exklusiv physische oder biotische Analogien bestimmte «Macht» rechtlich oder gar ethisch eingeschränkt werden kann.

15 Grabill, Rediscovering, s. Anm. 1, 177-179.
} 
Machtpolitik kann man nicht so loswerden, dass (natur)rechtliche Grundsätze aus dem Moralgesetz - etwa dem Dekalog - abgeleitet werden. Denn der Begriff des Stehlens im moralischen Prinzip «Du sollst nicht stehlen» setzt den juralen Aspekt der (Un-)Rechtoder (Un-)Gesetzmässigkeit bereits voraus: «Stehlen» bedeutet ja gerade unrechtmässiges Entwenden von Eigentum. Diese Terme wollen erst einmal rechtlich-historisch gefüllt werden, bevor sie der Spezifikation moralischer Pflichten oder Geboten dienen können.

Aus der Sicht des Gesetzgebers, schreibt Dooyeweerd, «the most important thing is to know what is to be understood by <unlawfully; and «natural law» cannot appeal to positive legal rules to find out what unlawfully means in the context of a principle that has not yet been positivized». ${ }^{16}$ Ohne Zweifel erschliessen sich im historischen Prozess der legalen Positivierung juraler Normen moralische Analogien, die zu einer theoretischen Vertiefung der ursprünglich undifferenzierten Bedeutung rechtlicher Begriffe führen - ohne dass diese jedoch ihren positiv-rechtlichen Sinn verlören und auf einmal moralisch würden! «[I]n a closed primitive jural order, the anticipating connection with morality - as expressed in the principles of equity, good faith, good morals, punishment according to guilt etc. - is absent. ${ }^{17}$ Die Dynamik der antizipierenden Erschliessung, die Dooyeweerd als grundlegenden, alle modalen Aspekte betreffenden Schöpfungsprozess versteht, wirft komplexe theoretische Probleme auf, die im naturrechtlichen Denkhorizont nur unzureichend erfasst werden können. In dieser Hinsicht verspricht Dooyeweerds, am theonomen Prinzip der Sphärensouveränität orientierter Denkansatz einigen Gewinn - und lässt sich nur von sehr weit weg betrachtet mit einer «reformierten»Verabschiedung oder Auszonung der cognitio dei naturalis aus dem reformierten Denken in Verbindung bringen.

- Dr. Johannes Corrodi arbeitet an einem SNF-Projekt zur Philosophie von Herman Dooyeweerd.

${ }^{16}$ Dooyeweerd, New Critique II, s. Anm. 10, 163.

17 Ders., New Critique I, s. Anm. 10, 29. 